\title{
Lysine Restriction Affects Feed Intake and Amino Acid Metabolism via Gut Microbiome in Piglets
}

\author{
Jie Yin ${ }^{a, b}$ Hui Han ${ }^{a, b}$ Yuying Lia,b Zhaojin Liuc Yurong Zhao ${ }^{c, d}$ Rejun Fang ${ }^{c, d}$

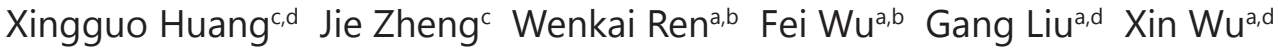 \\ Kai Wange Liping Sun ${ }^{e}$ Chunyong Li ${ }^{a}$ Tiejun Lia,d Yulong Yin ${ }^{a, d}$
}

aKey Laboratory of Agro-ecological Processes in Subtropical Region, Institute of Subtropical Agriculture, Chinese Academy of Sciences, Scientific Observing and Experimental Station of Animal Nutrition and Feed Science in South-Central, Ministry of Agriculture, Hunan Provincial Engineering Research Center for Healthy Livestock and Poultry Production, Hunan, 'University of Chinese Academy of Sciences, Beijing, 'Department of Animal Science, Hunan Agriculture University, Hunan, dHunan Co-Innovation Center of Animal Production Safety, Hunan, eInstitute of Apicultural Research, Chinese Academy of Agricultural Sciences, Beijing, China

\section{Key Words}

Lysine restriction $\cdot$ Microbiome $\cdot$ Feed intake $\cdot$ Piglet

\begin{abstract}
Background/Aims: Our previous reports suggested that dietary supplementation with lysine influenced intestinal absorption and metabolism of amino acids. In this study, we further investigated the effect of lysine restriction (30\%) on feed intake and we also tested the hypothesis that gut microbiome contributed to the potential mechanism of lysine restrictionmediated feeding behavior. Here, we profiled gut microbial communities by sequencing $16 \mathrm{~S}$ ribosomal ribonucleic acid ( $r R N A$ ) genes from gut samples as well as growth performance, serum hormones, and intestinal lysine transport in a piglet model. Results: Piglets preferred to the lysine restricted diet when giving three diets and the feed intake was markedly higher in the lysine-restricted group than that in the control group. Altered hormones (leptin, CCK, and ghrelin) might contribute to the feeding behavior caused by lysine restriction. Meanwhile, lysine transporting ability (SLC7A1 and SLC7A2 expression, intestinal electrophysiological changes, and amino acid pool in mesenteric vein) was decreased in response to lysine restriction. Through deep sequencing of bacterial rRNA markers, we observed that bacterial diversity was enhanced in the lysine-restricted group (Shannon $\mathrm{H}, \mathrm{PD}$, and Chao1). At the phylum level, lysine restriction enhanced gut Actinobacteria, Saccharibacteria, and Synergistetes abundances. At the family level, Moraxellaceae, Halomonadaceae, Shewanellaceae, Corynebacteriaceae, Bacillaceae, Comamonadaceae, Microbacteriaceae, Caulobacteraceae, and Synergistaceae

J. Yin and $\mathrm{H}$. Han contributed equally to this study

Tiejun Li

and Yulong Yin

Key Laboratory of Agro-ecological Processes in Subtropical Region,

Institute of Subtropical Agriculture, Chinese Academy of Sciences, Hunan (China)

E-Mail tjli@isa.ac.cn; yinyulong@isa.ac.cn
\end{abstract}

KARGER 
Yin et al.: Amino Acid and Microbiota Response to Dietary Lysine Restriction

abundances were increased in response to lysine restriction. Predictive functional profiling of microbial communities by PICRUSt also confirmed that dietary lysine restriction affected gut microbiome, which might further mediate amino acid metabolism, membrane transport, and endocrine system. Conclusion: Our results indicated that lysine restriction inhibited intestinal lysine transport and promoted feed intake, which might be associated with gut microbiome.

(C) 2017 The Author(s)

Published by S. Karger AG, Basel

\section{Introduction}

Dietary restriction is a promising therapeutic potential for preventing agingrelated diseases and extending healthspan via mediating feeding behavior and metabolic reprograming, especially for protein restriction [1-4]. Lysine serves as a key building block for protein synthesis and has been classified as an essential amino acid in humans and animals [5-7]. In previous studies, we used zein-formulated-lysine deficient diet and found that lysine deficiency inhibited feed intake and influenced intestinal absorption and metabolism of amino acids in mice and piglets $[8,9]$. This study was one of the first attempts to investigate if lysine limitation corresponds to the beneficial role of protein restriction.

Gut microbiota composition and activity can be rapidly shaped by dietary different nutritional level, nutrients, and texture. For example, a dry diet significantly improves oral bacterial diversity than wet-food diet in cat model [10]. Microbial mechanism underpinning the body of evidence indicates that various nutrients promote gut health and host metabolites and dietary factors facilitate the colonization of specific microbes [11]. Meanwhile, various organs are directly under the influence of bacterial metabolites that may further regulate physiological processes, such as endocrinology and feed intake regulation [12,13]. Microbial communities are now suspected of contributing to a myriad of epidemics of chronic diseases [14-17] and nutrients-mediated gut microbiome provides potential development of nutritional strategies for disease prevention and treatment. Currently, various hormones have been identified to serve as potential mechanism of gut microbes-controlling feed intake, such as leptin, glucagon-like peptide-1, Peptide YY, cholecystokinin (CCK), and ghrelin $[13,18,19]$. However, little evidence has concluded the effects of dietary lysine level on gut microbiome and its potential role in feeding behavior. Thus, in this study, we used $16 \mathrm{~S}$ ribosomal ribonucleic acid (rRNA) sequencing to investigate gut microbial diversity in lysine-restricted piglets.

\section{Materials and Methods}

\section{Animals and groups}

18 male piglets (Landrace $\times$ Large White) with average body weight $(21.26 \pm 0.39 \mathrm{~kg})$ were randomly divided into 6 triangular pens (Fig.1A) $(n=3)$ with three feeding troughs in each corner. Three lysine diets $(70 \%, 100 \%$, and 130\%) according to the NRC 2012 (Table 1) were fed in each trough and the feeding order and feeding troughs of diets were changed every day in a fixed order. Animals were free to drinking water and fed for 6 times per day for 25 days. Feed intake in each trough were recorded daily to evaluate the dietary preferences for diets varying in lysine concentration.

48 male piglets (Landrace $\times$ Large White) with average body weight were further bought to investigate the underlying mechanism in response to dietary different dosages of lysine. Animals were housed individually and assigned into two groups $(n=24)$ : a control group in which piglets received a basal diet according to the NRC 2012 (L100) and a lysine restricted group in which piglets were fed a diet containing $70 \%$ lysine diet (L70) of the control (Table 1). After 3 weeks (21 days), 8 piglets from L100 and L70 were randomly selected and killed for sample collection. Then 8 piglets from L100 group were fed the $70 \%$ lysine diet (L100.70) and the other 8 piglets still received the same 100\% lysine diet (L100.100). Similarly, the diet was changed into 100\% lysine for 8 piglets in L70 group (L70.100) and the other 8 piglets still received the same 70\% lysine diet (L70.70) for 3 weeks (Fig. 1D). Animals were free to drinking water and fed for 3 times per day. 
Blood leptin and gastrin determinations

All animals were anaesthetized with Zoletial 50 (Virbac Co., France) and then blood samples from mesenteric vein and carotid artery were harvested. Serum samples were separated from the blood after centrifugation at 3,000 $\times \mathrm{g}$ for $10 \mathrm{~min}$ and under $4{ }^{\circ} \mathrm{C}$. Leptin and gastrin were determined using ELISA kits in accordance with the manufacturer's instructions (Nanjing Jiangcheng Biotechnology Institute, China).

\section{Serum amino acids}

Serum samples from mesenteric vein were further used to test amino acid contents (His, Ser, Arg, Gly, Asp, Glu, Thr, Ala, Pro, Cys, Lys, Tyr, Met, Val, Ile, Leu, Phe, and Trp) by High-speed Amino Acid Analyzer L-8900 (Japan).

\section{Lysine transport}

The in vivo lysine transport was tested via Ussing chamber according to our previous report [20]. Briefly, 10 -cm segments were excised from the proximal jejunum and immediately placed in oxygenated HEPES-Tris buffer solution ( $\mathrm{pH} \mathrm{7.4)}$ at $37^{\circ} \mathrm{C}$. Mucosa sample were made for 4 replicates respectively, aperture area was set for $0.5 \mathrm{~cm}^{2}$ as the exposed tissue surface area, and the fluid volume in each chamber was $5 \mathrm{ml}$. Moreover, the concentration of lysine and mannitol was $0.2 \mathrm{mmol} / \mathrm{L}$ in the chamber buffer, respectively. The intestinal mucosal epithelium was mounted in an Ussing chamber (Physiological Instruments Inc., San Diego, CA). The mounted tissues were equilibrated for $10 \mathrm{~min}$ (baseline period) before the machine was adjusted to correct current and voltage according to the manual book (correction period, ensure that current and voltage value are zero, the transepithelial resistance (TR) is approximately $66 \mu \Omega / \mathrm{cm}^{2}$ ). One milliliter of a 0,1 , and 5 $\mathrm{mmol} / \mathrm{L}$ Lys solution was added to the luminal side of mucosa in a chamber, and mannitol solution was simultaneously added to the luminal side of serosa after equilibrating 10min (two half-chambers filled with $4 \mathrm{ml}$ HEPES-Tris buffer) to maintain the same osmotic pressure on both sides of the intestinal tissue. The changes in transepithelial resistance (TR) and short-circuit current (Isc) were recorded throughout the entire trial, which were used to evaluate the effect of Lys concentration on electrophysiological current in the intestinal mucosa of piglet. After $1 \mathrm{~h}, 2 \mathrm{ml}$ of solution from the mucosal and serosal sides were sampled into centrifuge tubes and stored at $-20^{\circ} \mathrm{C}$ for lysine analysis by an automated amino acid analyzer (L-8900, Hitachi, Japan). The changes in electrophysiological Isc $\left(\mu \mathrm{A} / \mathrm{cm}^{2}\right)$ and TR $\left(\mu \Omega / \mathrm{cm}^{2}\right)$ were calculated and lysine concentration of the diluted sample was determined.

\section{Real-time quantitative (RT-PCR)}

Total RNA from jejunum and ileum samples was isolated from liquid nitrogen frozen and ground tissues with TRIZOL regent (Invitrogen, USA) and then treated with DNase I (Invitrogen, USA) [21-23]. The reverse transcription was conducted at $37^{\circ} \mathrm{C}$ for $15 \mathrm{~min}, 95^{\circ} \mathrm{C} 5 \mathrm{sec}$. Primers used in this study were designed via Primer 5.0 according to mouse and pig gene sequence (Table 2 ). $\beta$-actin was chosen as the house-keeping gene to normalize target gene levels. The PCR cycling condition was 36 cycles at $94^{\circ} \mathrm{C}$ for $40 \mathrm{sec}, 60^{\circ} \mathrm{C}$ for $30 \mathrm{sec}$ and $72^{\circ} \mathrm{C}$ for $35 \mathrm{sec}$. The relative expression was expressed as a ratio of the target gene to the control gene using the formula $2^{-(\Delta \Delta C \mathrm{Ct})}$, where $\Delta \Delta \mathrm{Ct}=\left(\mathrm{Ct}_{\text {Target }}-\mathrm{Ct}_{\beta \text {-actin }}\right)_{\text {treatment }}-\left(\mathrm{Ct}_{\text {Target }}-\mathrm{Ct}_{\beta \text {-actin }}\right)_{\text {control }}$. Relative expression was normalized and expressed as a ratio to the expression in the control group [24-26].

\section{Isolation and characterization of microbiota}

Total genome DNA from ileal digesta was extracted using QIAamp DNA Stool Mini Kit and DNA concentration and purity was monitored on 1\% agarose gels. According to the concentration, DNA was 
Yin et al.: Amino Acid and Microbiota Response to Dietary Lysine Restriction

diluted to $1 \mathrm{ng} / \mu \mathrm{L}$ using sterile water. 16S rRNA genes of distinct regions (16SV3-V4) were amplified used specific primer with the barcode. All PCR reactions were carried out with Phusion $\AA$ High-Fidelity PCR Master Mix (New England Biolabs). Mix same volume of 1X loading buffer (contained SYB green) with PCR products and operate electrophoresis on $2 \%$ agarose gel for detection. Samples with bright main strip between 400-450bp were chosen for further experiments. Then, mixture PCR products was purified with Qiagen Gel Extraction Kit (Qiagen, Germany).

Sequencing libraries were generated using TruSeq® DNA PCR-Free Sample Preparation Kit (Illumina, USA) following manufacturer's recommendations and index codes were added. The library quality was assessed on the Qubit@ 2.0 Fluorometer (Thermo Scientific) and Agilent Bioanalyzer 2100 system. At last, the library was sequenced on an IlluminaHiSeq2500 platform and 250 bp paired-end reads were generated. Raw sequences are available in the NCBI SRA with accession numbers PRJNA376081.

\section{Microbiome analysis}

Paired-end reads were assigned to samples based on their unique barcode and truncated by cutting off the barcode and primer sequence. Paired-end reads were merged using FLASH (V1.2.7, http://ccb.jhu. edu/software/FLASH/), which was designed to merge paired-end reads when at least some of the reads overlap the read generated from the opposite end of the same DNA fragment, and the splicing sequences were called raw tags. Quality filtering on the raw tags were performed under specific filtering conditions to obtain the high-quality clean tags according to the QIIME (V1.7.0) quality controlled process. Sequences analysis were performed by Uparse software (Uparse v7.0.1001). Sequences with $\geq 97 \%$ similarity were assigned to the same OTUs. Representative sequence for each OTU was screened for further annotation. For each representative sequence, the GreenGene Database was used based on RDP classifier (Version 2.2) algorithmto annotate taxonomic information. In order to study phylogenetic relationship of different OTUs, and the difference of the dominant species in different samples (groups), multiple sequence alignment was conducted using the MUSCLE software (Version 3.8.31). OTUs abundance information were normalized using a standard of sequence number corresponding to the sample with the least sequences. Subsequent analysis of alpha diversity and beta diversity were all performed basing on this output normalized data. Alpha diversity is applied in analyzing complexity of species diversity for a sample through 6 indices, including Observed-species, Chao1, Shannon, Simpson, ACE, Good-coverage. All this indices in our samples were calculated with QIIME (Version 1.7.0) and displayed with R software (Version 2.15.3). Beta diversity analysis was used to evaluate differences of samples in species complexity, Beta diversity on both weighted and unweighted unifrac were calculated by QIIME software (Version 1.7.0). Cluster analysis was preceded by principal component analysis (PCA), which was applied to reduce the dimension of the original variables using the FactoMineR package and ggplot2 package in R software (Version 2.15.3). Principal Coordinate Analysis (PCoA) was performed to get principal coordinates and visualize from complex, multidimensional data. A distance matrix of weighted or unweighted unifrac among samples obtained before was transformed to a new set of orthogonal axes, by which the maximum variation factor is demonstrated by first principal coordinate, and the second maximum one by the second principal coordinate, and so on. PCoA analysis was displayed by WGCNA package, stat packages and ggplot2 package in R software (Version 2.15.3). Unweighted Pair-group Method with Arithmetic Means (UPGMA) Clustering was performed as a type of 


\section{Cellular Physiology Cell Physiol Biochem 2017;44:1749-1761

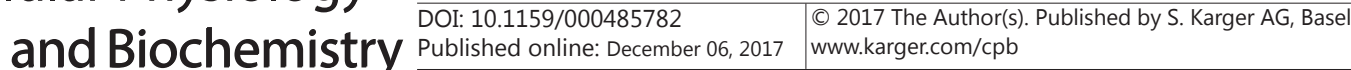

Yin et al.: Amino Acid and Microbiota Response to Dietary Lysine Restriction

hierarchical clustering method to interpret the distance matrix using average linkage and was conducted by QIIME software (Version 1.7.0).

\section{Predictive functional profiling of microbial communities}

OTUs were further used for genome prediction of microbial communities by PICRUSt (Phylogenetic Investigation of Communities by Reconstruction of Unobserved States) [27]. PICRUSt takes an input OTU table that contains identifiers that match tips from the marker gene (e.g. greengenes identifiers) with corresponding abundances for each of those OTUs across one or more samples. First, PICRUSt normalizes the OTU table by the $16 \mathrm{~S}$ copy number predictions so that OTU abundances more accurately reflect the true abundances of the underlying organisms. The metagenome is then predicted by looking up the pre-calculated genome content for each OTU, multiplying the normalized OTU abundance by each KEGG abundance in the genome and summing these KEGG abundances together per sample. The prediction yields a table of KEGG abundances for each metagenome sample in the OTU table. For optional organism-specific predictions, the per-organism abundances are retained and annotated for each KEGG. In this study, 6, 079 KEGG abundances were obtained and 5, 41, and 328 KEEG pathway annotations in KEEG level 1, 2, and 3 were identified in the Greengenes reference tree, respectively. We only focused on level 2 and 3 to investigate metagenome response to dietary lysine restriction.

\section{Statistical Analysis}

Data analysis were performed by using the one-way analysis of variance (ANOVA) to test homogeneity of variances via Levene's test and followed with student's T test [28](IBM SPSS 21.0 software). Data are expressed as the mean \pm SEN. Values in the same row with * or \# are significant $(P<0.05)$.

\section{Results}

Lys restriction promotes feed intake

Three lysine diets were provided in a triangular pen and piglets were free to these feeds (Fig. 1A). During the first two weeks, there was no obvious difference, while the feed intake and intake percent of diet containing 70\% lysine tended to increase from day 15 to day 24 (Fig. 1B and C), suggesting that piglets preferred to the lysine restricted diet. The results were further highlighted by that dietary lysine restriction (70\%) markedly enhanced feed intake compared with the control group (100\%) from 2-5 weeks ( $\mathrm{p}<0.05)$ (Fig. 1E). Meanwhile, feed intake from piglets fed the restricted diet was markedly lower after the diet changed into the control diet $(\mathrm{p}<0.05)$.

Intestinal microbiota of Lys restriction exhibits increased diversity and different composition

The hypervariable V3 and V4 regions of 16S rRNA genes were sequenced from ileal digesta and an average of 70, $910 \pm 10,651$ reads were generated from each sample. Binning sequences using a pairwise identity threshold of $97 \%$, we obtained an average of $433 \pm$ 100 operational taxonomic units (OTUs) per sample. Observed species, the estimators of community evenness (Shannon $\mathrm{H}$ ), and richness (Phylogenetic Diversity, PD, and chao1) were examined in alpha-diversity between the microbiomes (Fig. 2A-D). Although the longterm dietary $70 \%$ lysine tended to enhance ileal microbial diversity compared with the $100 \%$ lysine group, the difference was insignificant $(P D, 55.07 \pm 8.84 \mathrm{vs} .34 .82 \pm 3.09, p=0.056$; H, $3.69 \pm 0.29$ vs. $3.11 \pm 0.23$, $p=0.135$; chao $1,458.02 \pm 40.31$ vs. $360.32 \pm 32.39$, p=0.078) (Fig. 2). Once the dietary $100 \%$ lysine changed into $70 \%$ lysine (L100.70), a significant overall higher microbial diversity was noticed, with higher evenness $(H, 4.19 \pm 0.32$ vs. 3.11 \pm 0.23 , $\mathrm{p}=0.02$ ), and higher richness (PD, $50.55 \pm 1.95$ vs. $34.82 \pm 3.09$, $\mathrm{p}=0.003$; chao $1,491.78 \pm 40.51$ vs. $360.32 \pm 32.39, \mathrm{p}=0.02$ ) (Fig. 4). In contrast, the community richness was markedly decreased in the L70.100 group compared with the L70.70 group ( $<<0.05$ ) (Fig. 2).

To evaluate overall differences in beta-diversity between the microbiomes, we applied Principal Component Analysis (PCoA) to weighted and unweighted UniFrac distance metric 


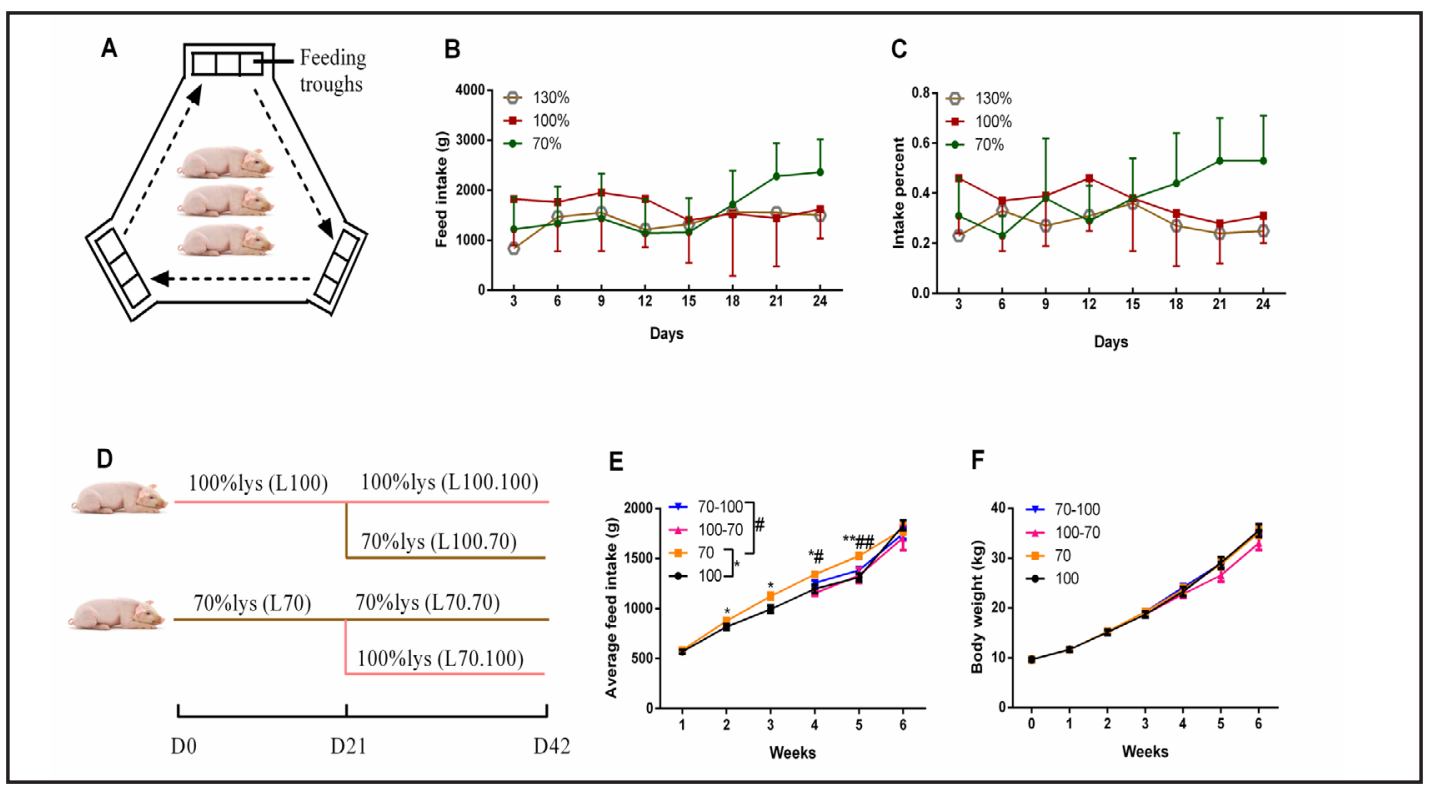

Fig. 1. Experimental design and growth performance. (A) Three pigs were fed in a triangular pen, with three feeding troughs in each corner. Feeding order and feeding troughs of diets were changed per day in a fixed order; (B and C) Average feed intake and intake percent of total intake (n=6); (D) Animal treatment and groups. More details can be found at the material section; ( $E$ and F) Average feed intake and body weight in response to dietary lysine restriction $(n=8)$. Data were expressed as the mean \pm SEN. * and \# mean the difference is significant $(\mathrm{P}<0.05)$.

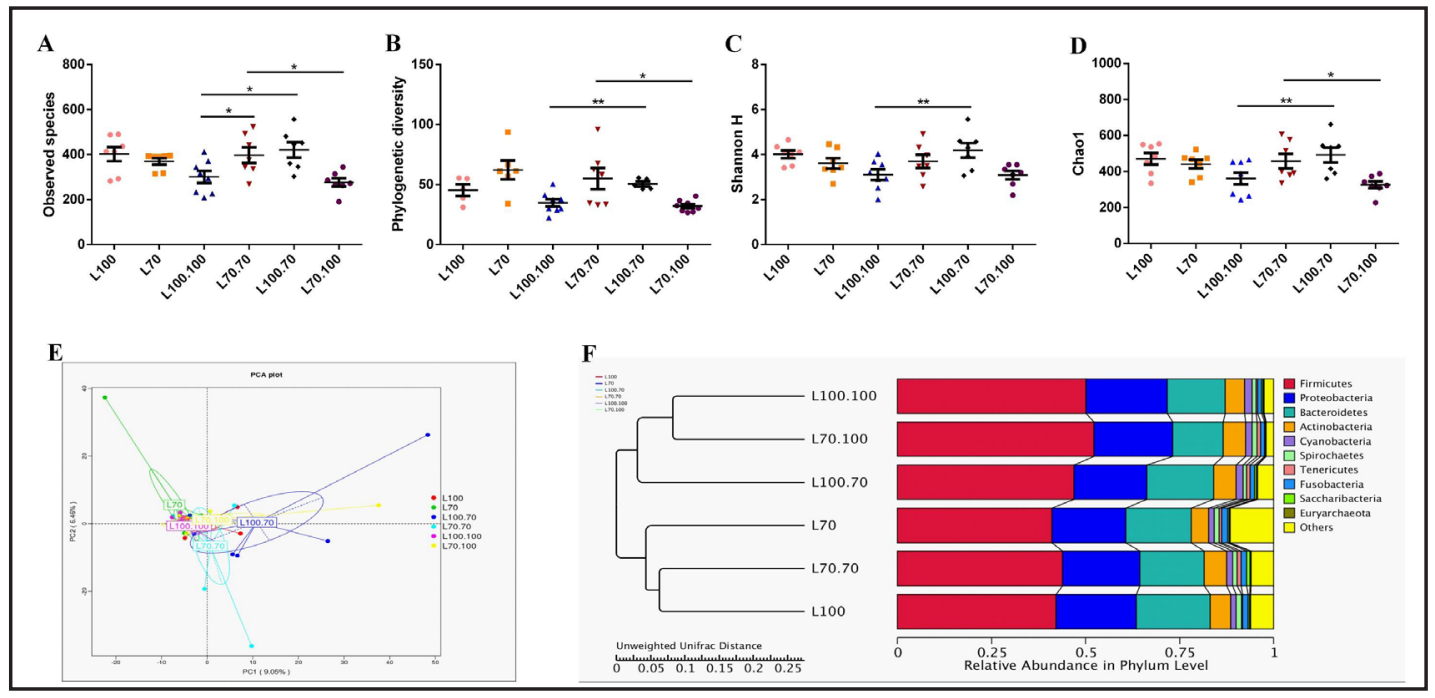

Fig. 2. Effects of lysine restriction on gut microbial diversity and unweighted UniFrac distances. (A) Observed species; (B) Phylogenetic Diversity (PD); (C) Shannon H index; (D) Chao1 index; (E) Principal Component Analysis (PCoA) of intestinal microbiomes; (F) Comparison of unweighted UniFrac distances between pairs of samples. Data were expressed as the mean \pm SEN $(n=8) . *$ means the difference is significant $(\mathrm{P}<0.05)$.

matrices generated for the sample set. Comparison of unweighted UniFrac distances between pairs of samples between different groups showed that the microbial community structure in dietary lysine restricted groups was more closely related to each other than to dietary normal feed (Fig. 2E and F).

The overall microbial composition for lysine restriction differed at the phylum and family levels (Fig. 3 and Table 3). The two largest phyla represented in each dataset 


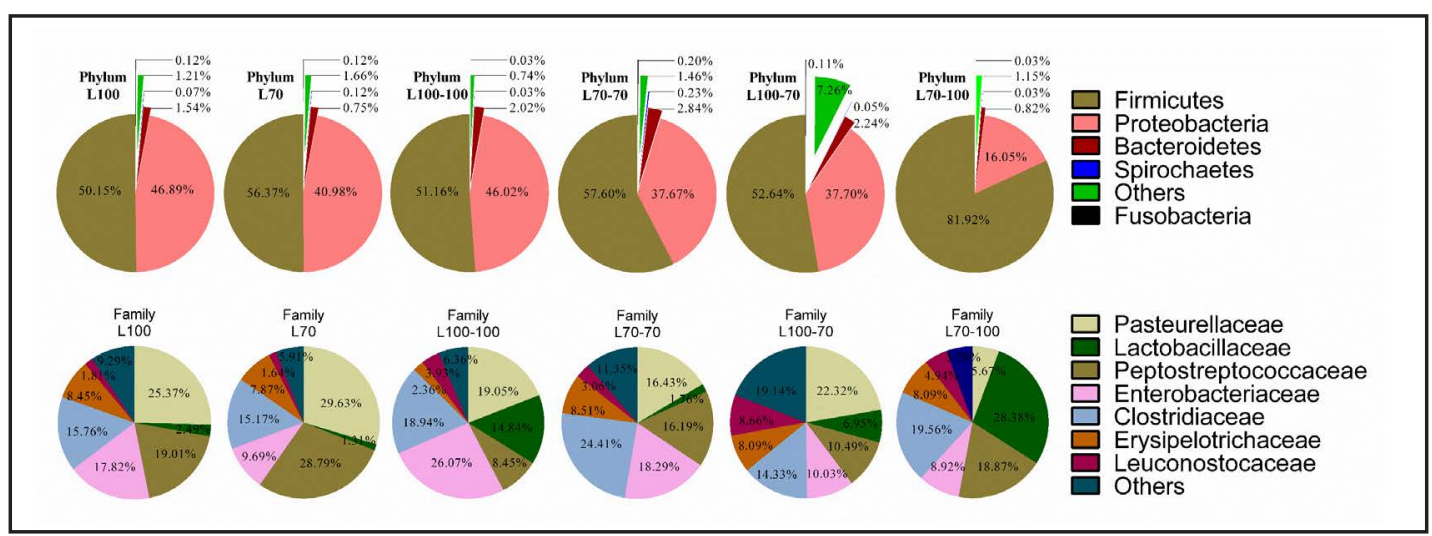

Fig. 3.16S RNA bacterial sequences represent in ileal samples. Pie charts of average values of relative abundance (percentage of sequences) of the most abundant bacterial groups: phylum and family found in the ileal microbiota $(\mathrm{n}=8)$.

Table 3. Listing of bacterial changes at the phylum and family levels. Note: $a,{ }^{*} 10^{-3} ; b,{ }^{*} 10^{-5}{ }^{*}$ means the difference was significant compared with the Lys100; \# means the difference was significant compared with the Lys70; \% means the difference was significant compared with the Lys100-100; \& means the difference was significant compared with the Lys70-70

\begin{tabular}{lcccccc}
\hline Item & \multicolumn{2}{c}{3 weeks } & \multicolumn{4}{c}{6 weeks } \\
\hline Phylum $^{\text {Lys100 }}$ & Lys70 & Lys100-100 & Lys70-70 & Lys100-70 & Lys70-100 \\
Actinobacteria $^{\mathrm{a}}$ & $2.6 \pm 1.2$ & $2.5 \pm 2.3$ & $1.3 \pm 0.7$ & $2.6 \pm 1.3 \%$ & $8.8 \pm 9.8$ & $2.8 \pm 3.4$ \\
Fusobacteria $^{\mathrm{a}}$ & $1.2 \pm 0.5$ & $1.3 \pm 0.9$ & $0.3 \pm 0.3$ & $2.0 \pm 2.2$ & $1.1 \pm 0.7 \%$ & $0.3 \pm 0.2$ \\
Saccharibacteria $^{\mathrm{b}}$ & $5.1 \pm 4.4$ & $4.8 \pm 3.6$ & $2.9 \pm 3.2$ & $15 . \pm 9.9 \%$ & $16.8 \pm 28.3$ & $11.8 \pm 31.0$ \\
Synergistetes $^{\mathrm{b}}$ & $1.2 \pm 3.2$ & $0.9 \pm 2.4$ & $0.3 \pm 0.7$ & $2.4 \pm 2.3 \%$ & $4.2 \pm 6.1$ & $0.0 \pm 0.0$ \\
Family & & & & & \\
Lactobacillaceae $^{\mathrm{b}}$ & $0.2 \pm 0.2$ & $0.1 \pm 0.1$ & $1.5 \pm 2.4$ & $0.2 \pm 0.1$ & $0.7 \pm 0.6$ & $2.8 \pm 2.3 \&$ \\
Moraxellaceae $^{\mathrm{a}}$ & $3.2 \pm 2.0$ & $2.0 \pm 1.3$ & $1.0 \pm 0.4$ & $3.5 \pm 1.6 \% \%$ & $11.6 \pm 16.9$ & $5.0 \pm 6.3$ \\
Halomonadaceae $^{\mathrm{a}}$ & $2.1 \pm 1.5$ & $1.9 \pm 0.8$ & $1.6 \pm 1.2$ & $3.5 \pm 1.6 \%$ & $10.5 \pm 16.6$ & $1.3 \pm 1.3 \&$ \\
Shewanellaceae $^{\mathrm{a}}$ & $1.3 \pm 0.8$ & $1.2 \pm 0.7$ & $1.1 \pm 0.7$ & $2.3 \pm 0.9 \%$ & $4.1 \pm 6.2$ & $1.0 \pm 1.3 \&$ \\
Corynebacteriaceae $^{\mathrm{a}}$ & $1.3 \pm 0.5$ & $1.3 \pm 0.8$ & $0.3 \pm 0.3$ & $0.7 \pm 0.3 \%$ & $2.8 \pm 2.9$ & $0.8 \pm 0.9$ \\
Bacillaceae $^{\mathrm{b}}$ & $13.8 \pm 19.5$ & $9.9 \pm 9.7$ & $7.4 \pm 3.9$ & $25.0 \pm 18.8 \%$ & $106.4 \pm 186.1$ & $27.4 \pm 42.1$ \\
Comamonadaceae $^{\mathrm{b}}$ & $87.8 \pm 74.4$ & $27.4 \pm 17.4$ & $12.9 \pm 9.2$ & $49.9 \pm 19.7 \% \%$ & $55.6 \pm 62.8$ & $25.5 \pm 17.6 \&$ \\
Microbacteriaceae $^{\mathrm{b}}$ & $9.3 \pm 10.2$ & $7.8 \pm 8.3$ & $4.0 \pm 4.4$ & $10.2 \pm 4.8 \%$ & $14.7 \pm 13.0$ & $18.2 \pm 30.6$ \\
Caulobacteraceae $^{\mathrm{b}}$ & $21.0 \pm 30.7$ & $6.0 \pm 4.9$ & $2.9 \pm 2.5$ & $8.4 \pm 4.9 \%$ & $12.6 \pm 11.6$ & $7.1 \pm 11.0$ \\
Synergistaceae $^{\mathrm{b}}$ & $1.2 \pm 3.2$ & $9.0 \pm 2.4$ & $0.3 \pm 0.7$ & $2.4 \pm 2.3 \%$ & $4.5 \pm 7.0$ & $3.2 \pm 3.7$ \\
Bacteroidaceae $^{\mathrm{b}}$ & $9.6 \pm 9.4$ & $7.8 \pm 11.6$ & $70.5 \pm 196.9$ & $8.1 \pm 6.8$ & $4.2 \pm 3.4$ & $0.5 \pm 1.5 \&$ \\
\hline
\end{tabular}

Firmicutes and Proteobacteria, while none of these differences were statistically significant after $\mathrm{t}$ test correction $(\mathrm{p}>0.05)$. Compared with the control diet, lysine restriction for 6 weeks markedly enhanced intestinal Actinobacteria, Saccharibacteria, and Synergistetes abundances $(\mathrm{p}<0.05)$ (Table 3). Diet changed into lysine restricted diet also increased the abundances of Actinobacteria, Saccharibacteria, and Synergistetes ( $\mathrm{p}>0.05)$. Fusobacteria was significantly enhanced after the diet changed into lysine restriction $(\mathrm{p}<0.05)$. At the family level, dietary lysine restricted diet for 6 weeks markedly enhanced intestinal Moraxellaceae, Halomonadaceae, Shewanellaceae, Corynebacteriaceae, Bacillaceae, Comamonadaceae, Microbacteriaceae, Caulobacteraceae, and Synergistaceae abundances $(\mathrm{p}<0.05)$, while Halomonadaceae, Shewanellaceae, and Comamonadaceae were reduced after the lysine restricted diet changed into the control diet (L70.100) $(p<0.05)$. These significant differences were further confirmed by LEfSe analysis, which uses linear discriminant analysis (LDA) coupled with effect size measurements to identify bacterial taxa whose sequences are differentially abundant in lysine restriction (data not show). 

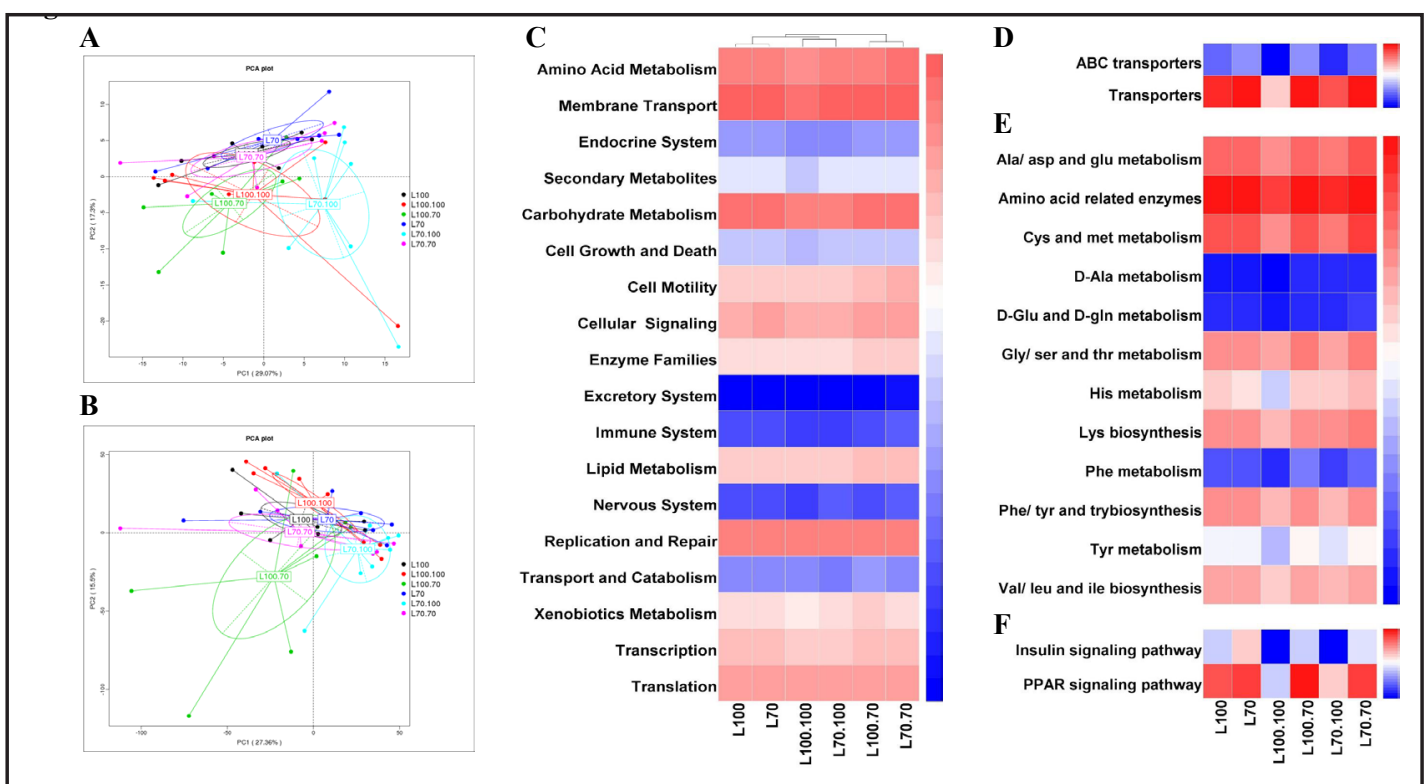

Fig. 4. Predictive functional profiling of microbial communities by PICRUSt. PCoA was generated in KEEG level 2 (A) and 3 (B); (C) KEEG pathway annotations in level 2 and amino acid metabolism mainly involved in lysine restriction; Membrane transport (D), amino acid metabolism (E), and endocrine system (F) were further analyzed in KEEG level 3 ( $n=8)$.

\section{Biofunction prediction of microbial communities}

In this study, PICRUSt was used to analyze functional profiling of microbial communities [27]. To evaluate overall differences in KEEG abundances between the metagenomes, PCoA was generated in KEEG level 2 and 3 (Fig. 4 A and B). The results showed that metagenome was highly regulated in response to dietary lysine fluctuation. In the level 2 (Fig. 4C), moderate dietary lysine restriction markedly affected cellular metabolism and physiology, such as amino acid metabolism, membrane transport, endocrine system, carbohydrate metabolism, cellular signaling, and replication and repair. For example, amino acid metabolism, membrane transport, and endocrine system were markedly enhanced in KEEG pathways after dietary a lysine-restricted diet (L70.70) (p<0.05). Membrane transport (Fig. 4D), amino acid metabolism (Fig. 4E), and endocrine system (Fig. 4F) were further analyzed in KEEG level 3 . In the membrane transporting system, transporters were significantly affected in response to dietary lysine restriction $(\mathrm{p}<0.05)$. Amino acid transporters mainly contribute to intestinal amino acid absorption, the KEEG analysis showed that dietary lysine restriction markedly influenced activities of amino acid-related enzymes and metabolism of Ala, Asp, Gly, Glu, Cys, Met, and His, and biosynthesis of lysine, Val, Leu, and Ile ( $\mathrm{p}<0.05)$.

\section{Lys restriction affects intestinal absorption of amino acids}

Our previous reports indicated that dietary different lysine concentration influences intestinal expression of lysine transporters, including SLC7A1 and SLC7A2. In this study, biofunction prediction of microbial communities suggested that dietary lysine restriction might mediate transporters and amino acid metabolism in piglets to regulate lysine absorption and metabolism. Thus, jejunal and ileal lysine transporters (SLC7A1 and SLC7A2) were determined via RT-PCR and the results showed that lysine restriction markedly downregulated intestinal SLC7A1 and SLC7A2 expressions ( $\mathrm{p}<0.05)$, which were increased when the diet has been changed into normal diet (L70.100) ( $p<0.05)$ (Fig. 5A-D).

The Ussing chamber used in the present study provided a physiologically relevant system for measuring the transport of lysine according to our previous reports. Although we failed to notice any significant difference in lysine transport (Table 4), Isc was obviously 


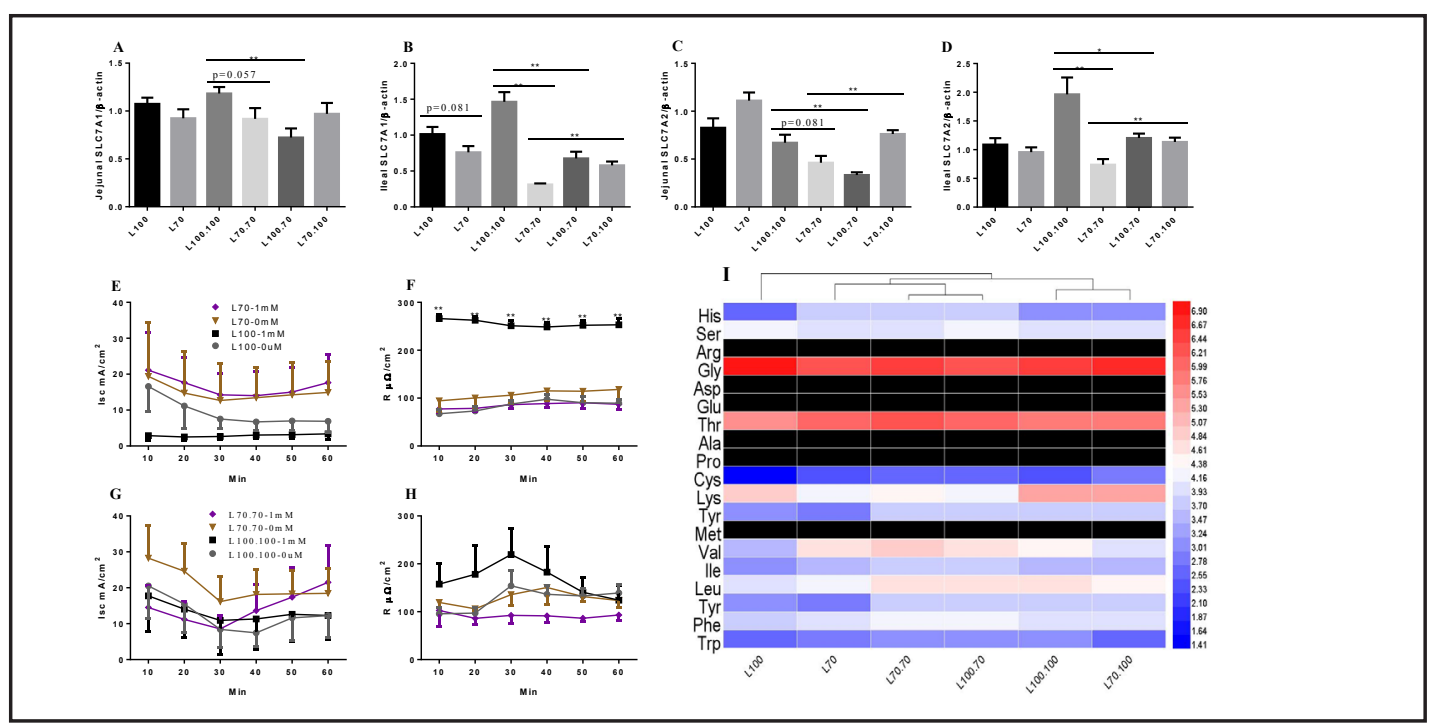

Fig. 5. Effects of lysine restriction on intestinal expressions of SLC7A1 and SLC7A2, electrophysiological changes, and amino acid pool. (A-D) mRNA abundances of SLC7A1 and SLC7A2 in the jejunum and ileum $(n=8)$; (E-H) Electrophysiological changes (Isc and R) measured at week 21 and 42 by Ussing assay $(n=4)$; (I) Heatmap of amino acids in mesenteric vein (the color is redder; the value is larger) $(\mathrm{n}=8)$. Data were expressed as the mean \pm SEN. * means the difference is significant $(\mathrm{P}<0.05)$.

higher and $\mathrm{R}$ was lower in lysine-restricted groups (Fig. 5E-H). Interestingly, $\mathrm{R}$ was markedly decreased in lysine-restricted group (L70) compared with the control (L100) (Fig. 5F). Electrophysiological changes in the lysinerestricted group further confirmed the lowered transporting ability of lysine in the intestinal mucosa after lysine restriction.

SLC7A1 and SLC7A2 contributed to the absorption of basic amino acids, such as lysine, His, and Arg. To investigate the absorption of amino acids from lumen, blood samples from mesenteric vein were collected and analyzed for amino acid concentrations (Fig. 5I). Lysine restriction markedly decreased serum lysine level $(\mathrm{p}<0.05)$, which was increased after the diet changed into the control diet (L70.100) $(\mathrm{p}<0.05)$. However, serum His exhibited an opposite trend that His increased in lysine restricted group and decreased in L70.100 group ( $p<0.05$ ). Glu, Gly, Cys, Ile, Met, Val, Lyr, Trp, Phe, and Pro were also influenced in this study, but further studies should be conducted to validate the potential mechanism.

\section{Lys restriction mediates satiety via influencing hormone}

Serum leptin and gastrin were further tested to investigate the mechanism of lysine restriction on feed intake (Fig. 6A-D). Although we failed to notice any significant difference in serum gastrin, lysine restriction tended to reduce leptin concentration in mesenteric vein $(\mathrm{p}=0.053)$. Leptin, a hormone made by adipose cells, helps to regulate feed intake 


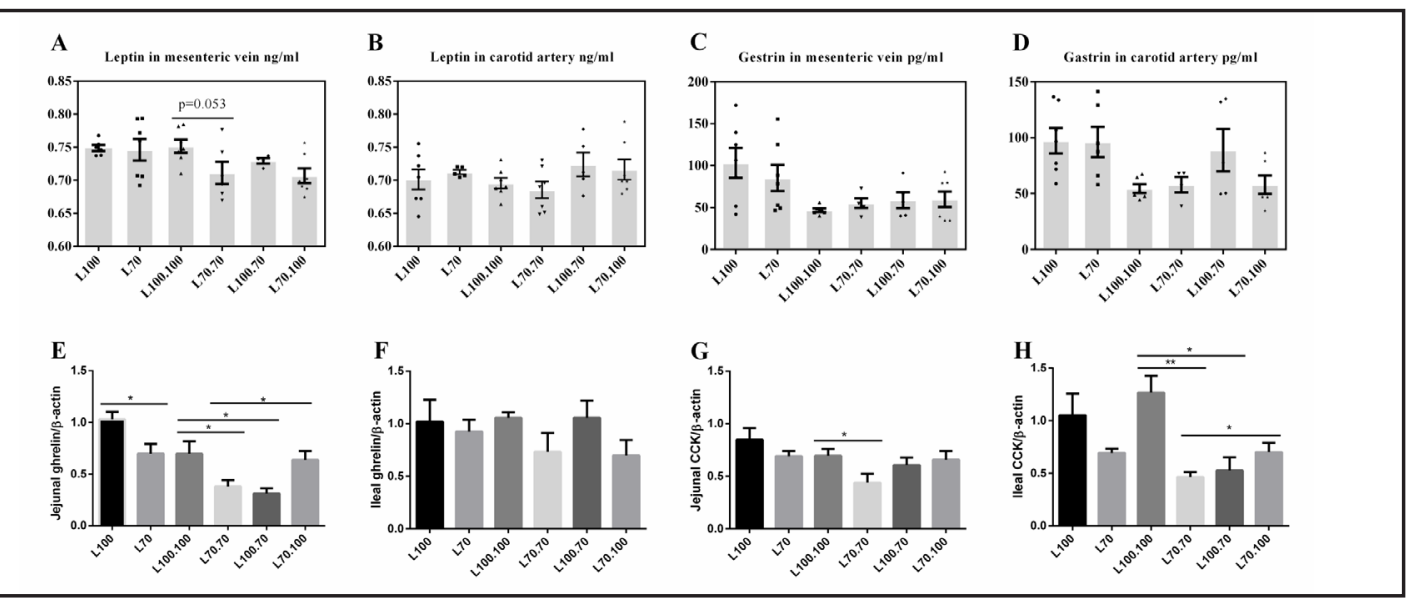

Fig. 6. Effects of lysine restriction on hormone system. (A and B) Serum leptin was determined by ELISA kit; (C and D) Serum gastrin was determined by ELISA kit; (E-H) Intestinal expressions of ghrelin and CCK were tested by RT-PCR. Data were expressed as the mean \pm SEN $(n=8)$. * means the difference is significant $(\mathrm{P}<0.05)$.

via inhibiting hunger [29] and the lowered leptin concentration in mesenteric vein was consistent with the higher feed intake in lysine restricted group.

We also determined intestinal CCK expression and the results showed that lysine restriction markedly downregulated intestinal CCK expression $(\mathrm{p}<0.05)$ (Fig. 6G and H). In addition, ileal CCK expression was decreased after the 100\% lysine diet (L100.100) changed into lysine restricted diet (L100.70) $(\mathrm{p}<0.05)$ and CCK was upregulated in L70.100 compared with the L70.70 group. Intestinal ghrelin was also tested and the result was similar to the expression of CCK $(\mathrm{p}<0.05)$ (Fig. 6E and F).

\section{Discussion}

Dietary preferences for feeds varying in lysine concentrations were firstly investigated and the results showed that piglets preferred to the lysine restricted diet. Meanwhile, feed intake was significant higher in the lysine-restricted group than that in the control group, which is contrast with the effect of protein restriction on feed intake [1]. These results concluded that lysine limitation failed to contribute to the merit of protein restriction.

Diet rapidly and reproducibly influences gut microbial compositions, structure, and metabolism [30-32], which may further control food intake via targeting hormones [13, 33]. In this study, we firstly reported that lysine restriction enhanced intestinal microbial community evenness (Shannon H) and richness (PD and chao1). Various reports indicated an involvement of the intestinal microbiome in growth performance [34, 35]. In this study, we observed significantly higher levels of the phyla Actinobacteria, Saccharibacteria, and Synergistetes in the lysine-restricted group. Actinobacteria are Gram-positive filamentous bacteria and serve as sources of novel antibiotics and secondary metabolites, which may be used in medicine or to improve growth and resistance to diseases [36]. Synergistetes exhibit Gram-negative characteristics and the ability to ferment amino acids [37]. We also found an increase in Moraxellaceae, Halomonadaceae, Shewanellaceae, Corynebacteriaceae, Bacillaceae, Comamonadaceae, Microbacteriaceae, Caulobacteraceae, and Synergistaceae at the family level in response to lysine restriction. Thus, these phyla response to lysine restriction might further mediate amino acid metabolism and feed intake.

PICRUSt is a novel approach to predict the functional composition of a metagenome using marker genes from 16S rRNA sequencing [27]. Using PICRUSt analysis, altered intestinal microbiome caused by lysine restriction might influence amino acid metabolism, membrane transport, endocrine system, carbohydrate metabolism, cellular signaling, and replication 


\section{Cellular Physiology Cell Physiol Biochem 2017;44:1749-1761 \begin{tabular}{l|l} 
DOI: 10.1159/000485782 & $\begin{array}{l}\text { O 2017 The Author(s). Published by S. Karger AG, Basel } \\
\text { www.karger.com/cpb }\end{array}$
\end{tabular} \\ Yin et al.: Amino Acid and Microbiota Response to Dietary Lysine Restriction}

and repair. Lysine transporters (SLC7A1 and SLC7A2), intestinal transporting ability, and serum amino acid pool were determined to confirm the role of lysine restriction on amino acid metabolism and membrane transport. Our results suggested that lysine restriction downregulated intestinal expression of SLC7A1 and SLC7A2, suggesting a lower transporting ability, which has been further confirmed by the Ussing chamber assay. Similarly, we also noticed that lysine deficiency for 4 and 7 days in mice inhibited intestinal SLC7A1 expression and protein abundances of CAT1 and CAT2 (encoded by the SLC7A1 and SLC7A2 genes) were decreased after IPEC-J2 cells exposure to lysine-free medium [8]. We further tested the amino acid pool in mesenteric vein and found that serum lysine was decreased and PICRUSt analysis predicted altered intestinal microbiome enhanced lysine biosynthesis in response to dietary lysine restriction. Histone is another basic amino acid along with lysine and the increased histone might compensate lowered lysine absorption. Meanwhile, dietary lysine restriction (L70.70 or L100.70) increased serum His, Cys, Val, and Ile abundances, which also validated the PICRUSt analysis that lysine restriction enhanced His, Cys, Val, and Ile metabolism and biosynthesis.

Endocrine system has been considered to be involved in the causality of gut bacteria and feed intake. For example, Everard et al. firstly reported that gut microbiota modulation improved leptin sensitivity and targeted enteroendocrine cell activity in obese and diabetic mice [38]. Furthermore, L-cells produced anorexigenic peptides (i.e. glucagon-like peptide-1 and Peptide YY), CCK, orexigenic hormones ghrelin, and short-chain acids/G-protein coupled receptors are also identified to serve as potential mechanism of gut microbes-controlling feed intake [13, 18, 19]. PICRUSt analysis predicted that dietary lysine restriction and altered intestinal microbiome might influence endocrine system, which further mediated feed intake in piglets. Leptin, a major satiety hormone of endocrine system, helps to regulate feed intake by inhibiting hunger [29]. In this study, we tested serum leptin concentrations in mesenteric vein and carotid artery and found that dietary lysine restriction tended to reduce serum leptin concentrations in mesenteric vein, indicating that lysine restriction associated with leptin abundance failed to inhibit hunger, which then enhanced feed intake in piglets. CKK also acts as a hunger suppressant and the mechanism for hunger suppression has been considered to be a decrease in the rate of gastric emptying [39]. In this study, we found that Lys restriction markedly downregulated intestinal CCK expression. Although we failed to investigate the effect of lysine restriction on gastric emptying, Baruffol et al. reported that L-lysine dose dependently delayed gastric emptying from 0-800 $\mathrm{mg}$ in rats and 0.5-7.5 $\mathrm{g}$ in humans [40]. Therefore, we speculated that lysine restriction inhibited the release and expression of satiety hormones (leptin and CCK) and promoted gastric emptying, which further enhanced feed intake in piglets.

\section{Conclusion}

Lysine restriction improved feed intake but failed to enhance body weight gain in piglet model. Expression of lysine transporters (SLC7A1 and SLC7A2) and transporting ability (Ussing chamber and amino acid pool) were reduced in response to lysine restriction. Altered hormones and gut microbiomes might contribute to the mechanism of feed intake regulation by lysine restriction.

\section{Acknowledgements}

This study was supported by the National Basic Research Program of China (973) (2013CB127301), Hunan Key Research Program (2017NK2320), National Natural Science Foundation of China (No. 31472106), and China Agriculture Research System (CARS-35).

We would like to thank Yao Jiming, Fang Wenjun, and Chen Yuquan from Guangdong Wangda group for the animal feeding support. We are grateful to the Public Service Technology Center, Institute of Subtropical Agriculture, Chinese Academy of Sciences for technical support. 


\section{Cellular Physiology Cell Physiol Biochem 2017;44:1749-1761 \begin{tabular}{l|l|l} 
and Biochemistry Published onlIne: December 06, 2017 & $\begin{array}{l}\text { (c) } 2017 \text { The Author(s). Published by S. Karger AG, Basel } \\
\text { www.karger.com/cpb }\end{array}$ \\
\hline
\end{tabular}}

Yin et al.: Amino Acid and Microbiota Response to Dietary Lysine Restriction

\section{Disclosure Statement}

The authors declare that they have no competing interests.

\section{References}

1 Mirzaei H, Suarez JA, Longo VD: Protein and amino acid restriction, aging and disease: from yeast to humans. Trends Endocrin Met 2014;25:558-566.

-2 Afsharmanesh M, Lotfi M, Mehdipour Z: Effects of wet feeding and early feed restriction on blood parameters and growth performance of broiler chickens. Animal Nutrition 2016;2:168-172.

3 Ciampolini M, Cecchi G: Dietary restriction to prevent fattening/insulin resistance/overall inflammation. Integr Food Nutr Metab 2017;4:1-2.

4 Lashinger LM, O’Flanagan CH, Dunlap SM, Rasmussen AJ, Sweeney S, Guo JY, Lodi A, Tiziani S, White E, Hursting SD: Starving cancer from the outside and inside: separate and combined effects of calorie restriction and autophagy inhibition on Ras-driven tumors. Cancer Metab 2016;4:18.

5 Bazer FW, Wang X, Johnson GA, Wu G: Select nutrients and their effects on conceptus development in mammals. Animal Nutrition 2015;1:85-95.

6 Smriga M, Mori M, Torii K: Circadian release of hypothalamic norepinephrine in rats in vivo is depressed during early L-lysine deficiency. J Nutr 2000;130:1641-1643.

7 Wu GY: Amino acids: metabolism, functions, and nutrition. Amino Acids 2009;37:1-17.

-8 Yin J, Li Y, Han H, Zheng J, Wang L, Ren W, Chen S, Wu F, Fang R, Huang X, Li C, Tan B, Xiong X, Zhang Y, Liu G, Yao J, Li T, Yin Y: Effects of Lysine deficiency and Lys-Lys dipeptide on cellular apoptosis and amino acids metabolism. Mol Nutr Food Res 2017;61:

-9 He LQ, Yang HS, Hou YQ Li TJ, Fang J, Zhou XH, Yin YL, Wu L, Nyachoti M, Wu GY: Effects of dietary l-lysine intake on the intestinal mucosa and expression of CAT genes in weaned piglets. Amino Acids 2013;45:383391.

10 Adler CJ, Malik R, Browne GV, Norris JM: Diet may influence the oral microbiome composition in cats. Microbiome 2016;4:

11 Gregory KE, Samuel BS, Houghteling P, Shan G, Ausubel FM, Sadreyev RI, Walker WA: Influence of maternal breast milk ingestion on acquisition of the intestinal microbiome in preterm infants. Microbiome 2016;4:

12 Evans JM, Morris LS, Marchesi JR: The gut microbiome: the role of a virtual organ in the endocrinology of the host. J Endocrinol 2013;218:R37-47.

13 Cani PD, Knauf C: How gut microbes talk to organs: The role of endocrine and nervous routes. Mol Metab 2016;5:743-752.

14 Stewart CJ, Embleton ND, Marrs ECL, Smith DP, Nelson A, Abdulkadir B, Skeath T, Petrosino JF, Perry JD, Berrington JE, Cummings SP: Temporal bacterial and metabolic development of the preterm gut reveals specific signatures in health and disease. Microbiome 2016;4:

15 Abuelfatah K, Zuki AB, Goh YM, Sazili AQ Abubakr A: Effects of feeding whole linseed on ruminal fatty acid composition and microbial population in goats. Animal Nutrition 2016;2:323-328.

16 Ciampolini M: A subjective, reproducible limit of intake in the child and the adult. Integr Food Nutr Metab 2016;3: 345-346.

17 Dominciano LC, Lee SH, Santello JM, Martinis ECD, Corassin CH, Oliveira CA: Effect of oleuropein and peracetic acid on suspended cells and mono-species biofilms formed by Staphylococcus aureus and Escherichia coli. Integr Food Nutr Metab 2016;3: 314-317.

18 Bauer PV, Hamr SC, Duca FA: Regulation of energy balance by a gut-brain axis and involvement of the gut microbiota. Cell Mol Life Sci 2016;73:737-755.

19 Mishra AK, Dubey V, Ghosh AR: Obesity: An overview of possible role(s) of gut hormones, lipid sensing and gut microbiota. Metabolism 2016;65:48-65.

20 He LQ, Yin YL, Li TJ, Huang RL, Xie MY, Wu ZL, Wu GY: Use of the Ussing chamber technique to study nutrient transport by epithelial tissues. Front Biosci-Landmrk 2013;18:1266-1275.

21 Liu M, Quek L-E, Sultani G, Turner N: Epithelial-mesenchymal transition induction is associated with augmented glucose uptake and lactate production in pancreatic ductal adenocarcinoma. Cancer Metab 2016;4:19. 


\section{Cellular Physiology Cell Physiol Biochem 2017;44:1749-1761 and Biochemistry Publisher $\begin{aligned} & \text { DOI: 10.1159/000485782 } \\ & \text { (c) } 2017 \text { The Author(s). Published by S. Karger AG, Basel } \\ & \text { www.karger.com/cpb }\end{aligned}$

22 Sprowl-Tanio S, Habowski AN, Pate KT, McQuade MM, Wang K, Edwards RA, Grun F, Lyou Y, Waterman ML: Lactate/pyruvate transporter MCT-1 is a direct Wnt target that confers sensitivity to 3-bromopyruvate in colon cancer. Cancer Metab 2016;4:20.

-23 Peck B, Schug ZT, Zhang Q Dankworth B, Jones DT, Smethurst E, Patel R, Mason S, Jiang M, Saunders R, Howell M, Mitter R, Spencer-Dene B, Stamp G, McGarry L, James D, Shanks E, Aboagye EO, Critchlow SE, Leung HY, Harris AL, Wakelam MJO, Gottlieb E, Schulze A: Inhibition of fatty acid desaturation is detrimental to cancer cell survival in metabolically compromised environments. Cancer Metab 2016;4:6.

24 Garcia-Recio EM, Pinto-Diez C, Perez-Morgado MI, Garcia-Hernandez M, Fernandez G, Martin ME, Gonzalez VM: Characterization of MNK1b DNA Aptamers That Inhibit Proliferation in MDA-MB231 Breast Cancer Cells. Mol Ther-Nucl Acids 2016;5:

-25 Chen KD, Huang KT, Lin CC, Weng WT, Hsu LW, Goto S, Nakano T, Lai CY, Kung CP, Chiu KW, Wang CC, Cheng YF, Ma YY, Chen CL: MicroRNA-27b Enhances the Hepatic Regenerative Properties of Adipose-Derived Mesenchymal Stem Cells. Mol Ther-Nucl Acids 2016;5:

-26 Iyombe-Engembe JP, Ouellet DL, Barbeau X, Rousseau J, Chapdelaine P, Lague P, Tremblay JP: Efficient Restoration of the Dystrophin Gene Reading Frame and Protein Structure in DMD Myoblasts Using the CinDel Method. Mol Ther-Nucl Acids 2016;5:

27 Langille MGI, Zaneveld J, Caporaso JG, McDonald D, Knights D, Reyes JA, Clemente JC, Burkepile DE, Thurber RLV, Knight R, Beiko RG, Huttenhower C: Predictive functional profiling of microbial communities using $16 \mathrm{~S}$ rRNA marker gene sequences. Nat Biotechnol 2013;31:814.

28 Malvi P, Chaube B, Singh SV, Mohammad N, Pandey V, Vijayakumar MV, Radhakrishnan RM, Vanuopadath M, Nair SS, Nair BG, Bhat MK: Weight control interventions improve therapeutic efficacy of dacarbazine in melanoma by reversing obesity-induced drug resistance. Cancer Metab 2016;4:21.

29 DiLeone RJ: The influence of leptin on the dopamine system and implications for ingestive behavior. Int J Obes (Lond) 2009;33 Suppl 2:S25-29.

-30 David LA, Maurice CF, Carmody RN, Gootenberg DB, Button JE, Wolfe BE, Ling AV, Devlin AS, Varma Y, Fischbach MA, Biddinger SB, Dutton RJ, Turnbaugh PJ: Diet rapidly and reproducibly alters the human gut microbiome. Nature 2014;505:559.

-31 Venkataraman A, Sieber JR, Schmidt AW, Waldron C, Theis KR, Schmidt TM: Variable responses of human microbiomes to dietary supplementation with resistant starch. Microbiome 2016;4:

-32 Hu JZ, Raikhel V, Gopalakrishnan K, Fernandez-Hernandez H, Lambertini L, Manservisi F, Falcioni L, Bua L, Belpoggi F, Teitelbaum SL, Chen J: Effect of postnatal low-dose exposure to environmental chemicals on the gut microbiome in a rodent model. Microbiome 2016;4:

33 Greiner TU, Backhed F: Microbial regulation of GLP-1 and L-cell biology. Mol Metab 2016;5:753-758.

-34 Myer PR, Wells JE, Smith TPL, Kuehn LA, Freetly HC: Cecum microbial communities from steers differing in feed efficiency. J Anim Sci 2015;93:5327-5340.

-35 Myer PR, Smith TPL, Wells JE, Kuehn LA, Freetly HC: Rumen Microbiome from Steers Differing in Feed Efficiency. Plos One 2015;10:

-36 Barka EA, Vatsa P, Sanchez L, Gaveau-Vaillant N, Jacquard C, Klenk HP, Clement C, Ouhdouch Y, van Wezel GP: Taxonomy, Physiology, and Natural Products of Actinobacteria. Microbiol Mol Biol Rev 2016;80:1-43.

-37 Bhandari V, Gupta RS: Molecular signatures for the phylum Synergistetes and some of its subclades. Anton Leeuw Int J G 2012;102:517-540.

-38 Everard A, Lazarevic V, Derrien M, Girard M, Muccioli GM, Neyrinck AM, Possemiers S, Van Holle A, Francois P, de Vos WM, Delzenne NM, Schrenzel J, Cani PD: Responses of Gut Microbiota and Glucose and Lipid Metabolism to Prebiotics in Genetic Obese and Diet-Induced Leptin-Resistant Mice. Diabetes 2011;60:2775-2786.

39 Gamble J, Kenny S, Dockray GJ: Plasminogen activator inhibitor (PAI)-1 suppresses inhibition of gastric emptying by cholecystokinin (CCK) in mice. Regul Pept 2013;185:9-13.

40 Baruffol C, Jordi J, Camargo S, Radovic T, Herzog B, Fried M, Schwizer W, Verrey F, Lutz TA, Steingoetter A: L-lysine dose dependently delays gastric emptying and increases intestinal fluid volume in humans and rats. Neurogastroenterol Motil 2014;26:999-1009. 